\title{
An annotated bibliography of Digital Scholarship in libraries
}

\author{
Carolyn Moritz \\ Vassar College Libraries \\ cmoritz@vassar.edu \\ in collaboration with \\ Florida State University Libraries \\ Office of Digital Research \& Scholarship
}

\section{Introduction}

The August 2017 release of De-Centering and Recentering Digital Scholarship: A Manifesto was the culmination of an ongoing conversation surrounding the work behind digital scholarship, scholarly communications, invisible labor, and the role of the library and the librarian within it. The manifesto represents voices at varying stages in their careers and a multitude of experiences, backgrounds, driving motivations, and dreams for the future. It also represents what Alison Mountz refers to as "painful differences and silences" in the voices not present that we yearn to include and illuminate. It emerged out of the need to frame digital scholarship's role in this moment as an emerging scholarship continuing the legacy of longstanding labor in critical and progressive librarianship. This bibliography, divided into Definitions of Digital Scholarship, Digital Scholarship in Practice, and Critical and Progressive Librarianship, provides a collaborative snapshot of many of the voices which have informed our theories and practices.

\section{Definitions of Digital Scholarship}

\section{Association of Research Libraries. (2016/2017). Digital Scholarship Support Profiles.} The Association of Research Libraries Digital Scholarship Support Profiles provided a curation of digital scholarship centers and initiatives across the U.S. These profiles have served as a useful benchmark for what peer institutions are doing to promote digital scholarship -based services. The growing network of digital scholarship activities across universities is a beneficial way for us to visualize the trends and changing natures of our work, as well as to identify potential avenues for research and collaboration.

Digital scholarship as an approach to practice, however, is outgrowing its nascent conceptualization as "digital tools and services." A summary review of the featured institutions in these Profiles and their definitions of digital scholarship provide an illustration of how both library workers and the broader academic community perceived digital scholarship centers at a specific point in time: 
"... identify innovative digital tools and resources that build on traditional research and teaching methods" (University of Oklahoma Digital Scholarship Laboratory).

"... apply digital research methods to humanistic or artistic material" (Temple University).

"... applying new technologies to advance the research and educational processes" (Case Western Reserve University).

These identified trends of "applying, "extending," and serving traditional modes of scholarship demonstrated the need for a standalone digital scholarship taxonomy so that library workers may more effectively articulate the work that they do and how it moves beyond a context of digital tools and services.

\section{Flanders, J. (2009). The productive unease of 21st-century digital scholarship. Digital}

\section{Humanities Quarterly. (3)3.}

The abstract for Productive Unease may be the most useful "short" definition of digital scholarship to date. Julia Flanders shifts the focus of the digital humanities away from specific tools or projects and towards an approach-based definition, paralleling the emergence of digital scholarship as a field beyond that of the service center. Digital scholarship, too, is "characterized at a deeper level by a more critical engagement with technology." Flanders' concept of "productive unease" calls out and champions the necessary tension that digital scholarly work presents to traditional notions of work in the academy.

The emphasis on intervention and productive unease provides an opportunity for active engagement with, and not just theoretical discussion of, a mode of work that refuses to take for granted concepts surrounding representation, medium, and structures. Here, still, traditional divisions of "scholars" from "technicians" prevail: statements such as "it is easier... to digitize an entire library collection" than to choose what is included, and "storage is cheaper than decision-making" reinforces a hierarchy of prioritized and valuable work. The prevalence of this contextual framing around development and maintenance in digital scholarship literature points to a need to critically examine our relationship with invisible labor and recognition of values-oriented infrastructure work.

\section{Kirschenbaum, M. G. (2010). What is Digital Humanities and what's it doing in English} departments? ADE Bulletin, 150, pp. 55-61.

As a critical discipline, digital scholarship looks to early discourse in the digital humanities and its evolving conversation around the definition and ideologies associated with the field. Kirschenbaum aligns digital humanities with "a common methodological outlook" as opposed to a narrower definition tied to texts or technologies. This contextualization of the digital humanities ("What's it doing in English departments?") challenges scholars to ask functional 
questions about what the work is, where it is, who does it, and why (the critical nature of both of our fields demands we also ask: where is the work not, who is not here, and why?).

\section{Digital Scholarship in Practice}

Ekstrøm, J., Elbaek, M., Erdmann, C., \& Grigorov, I. (2016, December 14). The research librarian of the future: Data scientist and co-investigator [Blog post].

The LSE Impact Blog asks what role the librarian plays as a research partner beyond that of "the data literate one." Authors Ekstrøm, Elbaek, Erdmann, and Grigorov envision an increasingly systems-level, holistic approach to academic research and, in particular, data science's capacity to facilitate a broader impact of research dimensions on one another across disciplines and variables. A rising focus on "applied" research positions in academia acknowledges the oft-superficial discipline barriers between issues in policy, sociology, economy, public health, geography, and environmental science. The authors' unique take on "tools-to-philosophy" approaches demonstrate a values-oriented practice around digital methodologies that must become a crucial component of the research librarian's future work. Digital scholarship finds itself both adding to and recognizing the value in deliberate development and design, from the systematic literature review enabling interdisciplinary mappings in existing research, to a linked open repository framework which allows for highly complex data evaluations.

\section{Fitzpatrick, K. (2011). Planned obsolescence: Publishing, technology, and the future of the academy. New York, NY: NYU Press. ${ }^{1}$}

Fitzpatrick's fundamental appraisal of the state of academic publishing in the digital sphere elevates the heart of digital scholarship practice. The decisions that surround the choice of medium, of assessment and review, of audience, and of the participation of the broader research community bring to the fore the significance of critically engaging with our methodologies as researchers and digital scholars. By retroactively discussing the process (and challenges) associated with publishing "Planned obsolescence" itself, Fitzpatrick's book provides an example of deliberately working values and ideals into the practice of day-to-day work beyond the finished (published) product.

The call to action here is not merely one born of a moral imperative: Fitzpatrick outlines a very tangible necessity to move past an unsustainable model of academic publishing, even and especially as that means challenging the entire concepts of completeness and authority on which the academic institution rests.

\footnotetext{
${ }^{1}$ From plannedobsolescence.net: "Planned Obsolescence was openly peer reviewed at MediaCommons Press in fall 2009; the draft version remains available online for open discussion."
} 


\section{Lippincott, J.K. \& Goldenberg-Hart, D. Digital scholarship centers: Trends \& good practice. Coalition for Networked Information Workshop Report.}

Joan Lippincott and Diane Goldenberg-Hart's compiled observations from a series of discussions on Digital Scholarship Centers (DSC's) begins with the logistical cases for a physical DSC in library spaces. In doing so, Trends \& Good Practice describes a physical, rather than merely conceptual, designation between digital scholarship and the digital humanities: the development of a DSC in the library democratizes the expertise and tools necessary for interdisciplinary collaboration outside of any single departmental burden. Drawing from the experiences of both large research institutions and liberal arts colleges, a close examination of the physical digital scholarship space yields revealing truths about the field of digital scholarship itself. Challenges around staffing, recruitment, and retention for programming and development positions, for instance, point to an ongoing tension between adaptability and sustainability. A contemporary scholarly institution requires both the infrastructure to change and plan according to quickly-altering contexts, as well as the capacity to work in terms of long-term preservation responsibilities.

CNI's report concludes that an adaptable and evolving skillset among all involved in a DSC is a higher priority than one single "type" of job candidate; this growing sentiment is a driving factor behind our assertion that digital scholarship spread from a discrete center to an integral part of training for all library workers. Many workshop participants echoed a shared commitment to provide more than a "tools and services" model and addressing necessary changes at the institutional level. Evaluation of digital scholarship work for promotion and tenure was a key part of this discussion, and participants from University of Nebraska-Lincoln shared their own Promotion \& Tenure Criteria for Assessing Digital Research in the Humanities.

\section{Padilla, T. (2016). Humanities data in the library: Integrity, form, access. D-Lib Magazine,} (22) $3 / 4$.

Values-oriented development, maintenance, and infrastructure work is the essential foundation of all meaningful digital scholarship. This detailed publication of concerns, assessments, discussions, collaborations, and derivatives surrounding infrastructure work shines a light on traditionally "invisible labor" and demonstrates a timely example of digital scholarship in practice. In this case, Thomas Padilla deliberately calls out libraries as information distributors to increase transparency of systems and organization practices. In understanding the decision-making process behind form, description, and accessibility of humanities data, Padilla illustrates the overlap between the [academic] library's obligation to its perceived users (other scholars), its central mission as a distributor of open information, and its provision of robust technical solutions that address long-term concerns while critically evaluating its own methodologies. 
Rumsey, A. S. (2011). New-model scholarly communication: Road map for change. Scholarly Communication Institute, (9).

The Scholarly Communication Institute's 2011 "Road Map for Change" resulting from three days of discussions at the University of Virginia Library provides an exemplary answer to the question of "what Digital Scholarship does" and an illustration of meaningful discourse in practice. It is worth noting that several of these participants have been included elsewhere in this bibliography, including Kathleen Fitzpatrick and Julia Flanders. SCI Director Abby Smith Rumsey outlines the conclusive "next steps" and relevant actors that came out of these discussions with a useful conceptual link between digital scholarship and new-model scholarly communication, defining the latter as the product of putting the practices of digital scholarship "into the processes of production, publishing, curation, and use of scholarship."

As of 2011, participants' actionable priorities included experimentation in multimedia publishing, expanding open access and fair use with practical business models for lost revenue compensation, reevaluation of assessment and credit for both graduate theses and advanced scholarly output, and new sources of funding and support. These road map guidelines provide a useful benchmark for assessing the current work of libraries and digital scholarship practitioners in 2017. This perspective yields a promising vision of the evolution of the work that continues to be done, and an argument against the introspective and unhelpful pressure to define digital scholarship in order to prove its existence as a field and mode of practice. Rumsey is also the author of "When We Are No More: How Digital Memory is Shaping our Future," and puts a number of these guidelines into practice in her own scholarly work in the realm of mindful technical development and new-mode scholarly communication.

\section{Critical and Progressive Librarianship}

\section{Almeida, N. (2017). Open educational resources and rhetorical paradox in the neoliberal univers(ity). Journal of Critical Library and Information Studies, (1)1.}

The resounding takeaway from the "Decentering" manifesto's ten theses is that digital scholarship work must, at its heart, remain critical, mindful, and continually self-aware. Open production and dissemination of scholarship is the core priority of both those under the various "open" banners and of libraries as a whole. In our work with faculty and researchers we continue to recognize a tension between the goal of advancing scholarship and distributing knowledge that drives many who choose a career in the academy, and the real and immediate requirements for grant funding, promotion and tenure, and labor valuation.

Nora Almeida's cautionary words are not an obstacle to the promotion of Open Educational Resources (OER) but indeed a grounded vehicle through which "a discourse of both critique and possibility" can be both possible and meaningful. We work with the firm conviction that higher 
education and resource production require, at an institutional level, far more attention than any one utopian vision can resolve; that the core questions behind that attention include "Who is missing from this discussion?", "Whose labor is behind this output?", "Who decides what scholarship is valuable and what labor is necessary?" Unearthing the answers to these questions means not simply sitting at a table of like-minded individuals and conceiving of solutions in a vacuum, but rather putting forth the effort to find and promote the most highly-impacted groups and individuals who are already engaging in this discourse, producing this labor, and driving the goals of digital scholarship and critical librarianship forward.

\section{Lovins, D. (2017). Broken-world vocabularies. D-Lib Magazine, (23)4.}

At the intersection of invisible labor and digital scholarship is the discourse surrounding technical, maintenance, development, and infrastructure work. Digital scholarship relies on a technical infrastructure that makes new initiatives and projects possible. Critical evaluation of information as a public good relies on the means of displaying, disseminating, assessing, and valuing information in a digital context. Asserting the role of the librarian as a collaborator and research partner by virtue of expertise in digital technologies, information networks, and infrastructure relies on the aforementioned resources to exist in the first place. The labor behind technical infrastructure is not merely a service but an end to itself; development in the scholarly communications and digital scholarship realm is value-driven and those who conduct this labor have expertise and research interests of their own.

One major example of this value-driven development is that of the Semantic Web, the overlap between digital scholarship values and the goals behind realizing organized information as relational and networked and, fundamentally, open. The future of the Semantic Web relies on information and data's openness in order to function at its full potential; the work of developers and maintainers here is inherently non-neutral and those maintaining this infrastructure have a stake in the future of open data as a key component of their labor operating successfully. Daniel Lovins and Diane Hillmann's emphasis on a "broken-world thinking" provides a vocabulary in which the labor of maintenance and repair is not a problem to be cured but rather an integral part of a living, dynamic web. The work here acknowledges the power of language itself to shape how work is recognized and conceptualized, and returns agency to the hands of the aforementioned "maintainers" by providing a language with which to relate the significance of their work to the broader values of digital scholarship. This same recognition of the significance of language and a deliberate vocabulary is what drives TaDiSAIL, the taxonomy of digital scholarship labor.

Mountz, A., Bonds, A., Mansfield, B., Loyd, J., Hyndman, J., Walton-Roberts, M., Basu, R., Whitson, R., Hawkins, R., Hamilton, T., \& Curran, W. (2015). For slow scholarship: A 
feminist politics of resistance through collective action in the neoliberal university. $A C M E$ : An International Journal for Critical Geographies, 14(4), 1235-1259.

The deliberate structure of both the manifesto and this bibliography reflect a desire to reach into theory and discourse and pull out of it tangible practices and ways of working. Alison Mountz, Anne Bonds, Becky Mansfield, Jenna Lloyd, Jennifer Hyndman, Margaret Walton-Roberts, Ranu Basu, Risa Whitson, Roberta Hawkins, Trina Hamilton, and Winifred Curran's work represent the fruits of the Great Lakes Feminist Geography Collective, advocating a feminist ethics of care in scholarship that demands scholars do not merely "make more time" in the productive sense but, in fact, change time (emphasis in original) in order to bring self-care and care for others "out of hiding in private times and spaces" and into the open as a real and necessary part of the life of a researcher, student, and laborer in academia.

Emphasizing slow and deliberate scholarship to produce high-quality, meaningful, and careful output includes rethinking the goals and structures of the neoliberal university framework and to "count what others don't" using a reassessment of how we evaluate scholarship and an alternative metrics framework for recognizing diverse types of impact.

Trosow, S.E. (2014/2015). The commodification of information and the public good: New challenges for a progressive librarianship. Progressive Librarian, (43), pp. 17-29.

It is essential to acknowledge and reaffirm the tradition of progressive librarianship on which digital scholarship stands. Alongside others, Samuel Trosow reminds librarians of the library's critical role in the public sphere and the nature of information as a public good, reasserting the statement of the Progressive Librarians Guild that "we strongly oppose the commodification of information which turns the 'information commons' into privatized, commercialized zones." Trosow's work is a call to action to not just oppose the intellectual property regime but to consider alternatives that meaningfully reward creators while de-emphasizing market-driven goals; to resist "the pressure to conform to the model of the market place," and to question the organization of library governance as a mirror of corporate managerial frameworks. 